\title{
Des décisions parisiennes aux municipalités cantonales : la mise en œuvre de la politique directoriale dans le département de l'Aisne
}

\section{OpenEdition}

Journals

Édition électronique

URL : https://journals.openedition.org/ahrf/774

DOI : $10.4000 /$ ahrf.774

ISSN : 1952-403X

Éditeur :

Armand Colin, Société des études robespierristes

Édition imprimée

Date de publication : 1 décembre 2002

Pagination : 115-133

ISSN : 0003-4436

Référence électronique

Laurent Brassart, «Des décisions parisiennes aux municipalités cantonales : la mise en œuvre de la politique directoriale dans le département de l'Aisne ", Annales historiques de la Révolution française [En ligne], 330 | octobre-décembre 2002, mis en ligne le 17 avril 2008, consulté le 23 avril 2022. URL :

http://journals.openedition.org/ahrf/774 ; DOI : https://doi.org/10.4000/ahrf.774 
D E S D É C I S I O N S P A R I S I E N N E S

\author{
A U X M U N I C I P A L I T É S C A N T O N A L E S : \\ LA M ISE EN CEUVRE DE LA POLITIQUE \\ DIRECTORIALE DANS LE DÉPARTEMENT DE L'A ISNE
}

\title{
LAURENT BRASSART
}

\begin{abstract}
Le fonctionnement du système des pouvoirs locaux dans le département de l'Aisne a évolué au cours du Directoire. En l'an IV, I'hétérogénéité politique du personnel local, loin de rendre inefficace l'application des décisions parisiennes l'a, au contraire, renforcée. En revanche, le coup d'État du 18 Fructidor an $\mathrm{V}$, qui s'est caractérisé dans le département par l'élimination des opposants au régime de tous les pouvoirs locaux importants, a paradoxalement affaibli le poids de l'État et de son centre décisionnel parisien. L'homogénéité politique du nouveau personnel a contribué à l'élaboration des solidarités locales et à une volonté toujours plus affirmée d'autonomie du pouvoir local face au centre parisien. De nouvelles règles du jeu entre Paris et la province se reformulent, consacrant la médiation politique et sociale d'une notabilité départementale en formation.
\end{abstract}

Mots clés: Aisne; administration départementale; personnel politique ; emprunts forcés ; canton de Bohain.

Étudier la circulation de l'information et la prise de décision entre Paris et la province sous le Directoire revient à considérer le rapport Paris/province comme un jeu toujours en recomposition entre les rapports de force politique locaux et les impulsions nationales (1). C'est pourquoi, dans le cadre de l'Aisne, un département picard modéré, qui n'a jamais été considéré par le pouvoir parisien révolutionnaire comme présentant des risques de fédéralisme ou d'hostilité, la recomposition du rapport Paris/province peut être appréhendée au moyen, d'une part, de l'exécution

(1) Cf. Roger DUPUY (ss dir), Pouvoir local et Révolution. La frontière intérieure, Rennes, Presses Universitaires de Rennes, 1995,570 p.

Annales historiques de la Révolution française - 2002 - $N^{\circ} 4\left[\begin{array}{lll}115 & \text { à } & 133\end{array}\right]$ 
locale des décisions nationales, d'autre part, par la marge d'autonomie et la capacité d'initiative dont sont investis les différents acteurs du pouvoir local. L'étude de la levée des emprunts forcés et du maintien de l'ordre public dans le canton de Bohain, alors le plus troublé du département en l'an IV et l'an VII nous permettra d'illustrer ces rapports Paris / province. Cependant, entre l'an IV, qui inaugure la mise en place des pouvoirs locaux définis lors de la Constitution de l'an III, et l'an VII, qui sera, au regard de l'histoire, leur ultime année de fonctionnement, bien des paramètres locaux et nationaux, et parfois des révolutions silencieuses, ont fait évoluer le régime directorial. L'entreprise de stabilisation du régime s'est accompagnée localement de multiples tâtonnements pour redéfinir le lien politique entre l'État et les citoyens. L'analyse des relations de pouvoir entre les différents niveaux et les différents acteurs du système des pouvoirs locaux et le centre parisien suppose la prise en considération d'une dimension inter-personnelle du pouvoir (2). L'attitude des administrateurs et du commissaire départementaux, les liens interpersonnels faits de solidarité et de domination qu'ils établissent avec l'ensemble des acteurs du système des pouvoirs de l'État seront au centre de cette étude.

\section{Circulation de l'information et prise de décision politique sous le Premier Directoire. L'exemple de l'an IV.}

Exécuter les directives du pouvoir central parisien en l'an IV passe notamment pour l'administration départementale par l'accomplissement sans faille du devoir fiscal.

Afin de rétablir le cours de l'assignat, le Directoire avait proposé aux Conseils un emprunt forcé. Celui-ci, voté le 19 frimaire an IV (9 décembre 1795), avait pour fonction de retirer de la circulation le plus grand nombre d'assignats possible dans les délais les plus courts. Les travaux de répartition et de perception incombaient entièrement au nouveau système des pouvoirs locaux mis en place en vendémiaire et brumaire an IV (3). Cet emprunt devait exclusivement reposer sur le quart des citoyens les plus fortunés du département, qu'il convenait de répartir entre seize classes numériquement égales, en fonction de la valeur de leur fortune. Ces classes étaient établies par l'administration départementale après que les municipalités cantonales lui aient fait parvenir les rôles fiscaux des communes les composant. Une

(2) Cf. Gérard NOIRIEL, État, nation et immigration. Vers une histoire du pouvoir, Paris, Belin, 2001, 400 p. ; Pierre GREMION, Le pouvoir périphérique. Bureaucrates et notables dans le système politique français, Paris, Le Seuil, 1976, 478 p. ; Bulletin de la SHMC, « Entre pouvoirs locaux et pouvoirs centraux : figures d'intermédiaires », 1998/3 et 1998/4, pp. 1-86.

(3) Dans le département de l'Aisne, l'administration départementale a été installée le 28 vendémiaire an IV, les assemblées municipales de canton le 24 brumaire an IV. Quant au commissaire départemental, il a pris ses fonctions le 19 frimaire an IV. 
fois le travail de répartition des citoyens entre les classes achevé par l'administration départementale, il était retourné aux administrations cantonales afin qu'elles entreprennent les opérations de recouvrement.

En vertu des lois des 18 et 19 frimaire an IV (8 et 9 décembre 1795), l'emprunt forcé aurait dû être recouvré dans tous les départements de la République pour le 15 nivôse an IV (5 janvier 1796) et soldé pour le mois de pluviôse an IV (4). Or dans l'Aisne, c'est seulement à la date du 8 ventôse an IV (27 février 1796) que les opérations de répartition ont été achevées (5). Pour ce qui est du recouvrement, il ne concerne à cette date que les cinq classes les plus imposées du département. Les onze autres, ayant été dressées tant bien que mal ce jour même. Au bout du compte, l'administration départementale a donc un retard de plus de trois mois sur les délais légaux prescrits. Et encore faut-il préciser que la levée n'est que partielle.

Pourquoi un tel retard? Le premier obstacle rencontré dans l'exercice des fonctions fiscales par l'administration départementale et son commissaire tient à la résistance des communautés locales. Le refus d'établir des rôles, de les remettre vierges de toute irrégularité à l'administration départementale, n'est pas un devoir qui va de soi pour des citoyens quelque peu habitués à s'accommoder depuis 1789 d'un «État sans fisc » (6). Plus la municipalité est rurale, moins l'obéissance fiscale est aisée (7). Tout fonctionne comme si, face à l'agression de l'État, la communauté rurale réagit encore collectivement quand la ville laisse libre cours à une individualisation des attitudes fiscales. Pour vaincre ces résistances, l'administration est contrainte de perdre un temps précieux en dépêchant localement des garnisons de gendarmerie : tantôt pour se saisir manu militari de l'agent en possession des rôles de sa commune, tantôt pour rétablir l'ordre là où les malcontents se font menaçants (8). Tel est notamment le cas dans le petit bourg rural de Vervins peuplé d'environ 2700 habitants. Le 27 nivôse (17 janvier 1796), des imposés s'opposent violemment à la municipalité contestant l'impartialité des rôles qu'elle a dressés. La municipalité réclame dans l'urgence à l'administration départementale l'envoi d'un détachement de gendarmerie pour rétablir le calme tout en précisant «qu'on essuiera

(4) Loi du 19 frimaire an IV. Art. VIII : « les rôles seront mis en recouvrement avant le 15 nivôse prochain par les percepteurs des contributions directes... »; Art. IX : «Les sommes seront exigibles un tiers avant la dernière décade de nivôse, et le surplus en pluviôse suivant. »

(5) Archives départementales de l'Aisne, L 892, 8 ventôse an IV.

(6) Cf. Claudine WOLIKOW, «Fiscalité et citoyenneté sous le Directoire ou la déroute de l'État sans fisc » dans Hervé LEUWERS et alii (éd.), Du Directoire au Consulat, t. 2, L'intégration des citoyens dans l'ÉtatNation, Lille, 2000, pp. 217-237. an IV.

(7) ADA, L 892, lettre de l'administration centrale de l'Aisne au ministre des Finances, le 28 nivôse

(8) Ibid., L 375, 25 nivôse an IV. L'administration départementale donne ordre à la gendarmerie d'arrêter le président de l'administration cantonale de Gandelus «puisque aucune opération n'est effectuée dans le canton. » 
beaucoup de difficultés pour [le] recouvrement»(9). Quinze gendarmes furent envoyés à Vervins où ils stationnèrent, une quinzaine de jours, jusqu'au 13 pluviôse an IV (2 février 1796) (10).

Cette résistance fiscale, qui n'apparaît cependant pas comme l'attitude de la majorité des municipalités de canton, outre le fait qu'elle retarde les travaux de répartition de l'emprunt, compromet aussi son équité. Acculée, par le pouvoir central parisien, à hâter les opérations de l'emprunt, l'administration n'a ni le temps ni la volonté d'entreprendre une révision scrupuleuse des rôles remis par les administrations municipales (11). La porte est ouverte pour que les injustices soient entérinées. Le commissaire départemental Joachim Dormay avertit le ministre des Finances que : «Dans quelques-unes [des communes], les agents sont accusés de partialité et d'assez peu de délicatesse pour s'être portés à un taux infiniment au-dessous du seuil de celui qu'ils auraient dû être placés. On prétend même que la diversité des positions sur la Révolution y est entrée pour quelque chose » (12). Dans certaines municipalités cantonales, le républicain est une cible toute désignée pour l'effort fiscal nécessaire à la survie du régime dont il est le partisan, et ce pour le plus grand profit du contre ou de l'anti-révolutionnaire. Les contribuables les plus républicains sont alors placés dans une classe fiscale largement supérieure à celle qui correspond à leur fortune, pendant que d'autres, plus connus pour leur opposition au régime, jouissent du bonheur fiscal de voir leurs biens sous-évalués. Un autre cas d'injustice se manifeste lorsqu'une municipalité plutôt acquise au régime, applique strictement, voire avec zèle, les instructions légales. En confiant aux administrations locales, la rédaction des rôles fiscaux, l'État a pris le risque d'étendre les inégalités fiscales entre cantons. À fortune égale, un citoyen risque d'être davantage imposé dans un canton pro-républicain que dans un autre contrerévolutionnaire. Telle est la signification de l'émeute qui avait secoué le bourg rural de Vervins le 27 nivôse an IV (17 janvier 1796). Une fois le calme rétabli avec le stationnement d'un détachement de la gendarmerie, les quarante et un Vervinois assujettis à l'emprunt décident alors d'en référer au ministre des Finances, par la médiation d'une adresse. Ils se plaignent d'être surtaxés, surtout en comparaison des autres communes du département : «Ceux portés au département par la municipalité de cette commune pour servir à asseoir l'imposition des imposables avoient fixés les fortunes plutôt au-dessus qu'en dessous de leurs valeurs réelles; tandis qu'il

(9) Ibid., 25 nivôse.

(10) Ibid., 13 pluviôse an IV.

(11) Ibid., L 439, 26 nivôse an IV, le commissaire départemental au ministre des Finances : «Les courts délais qui sont accordés ne permettent pas à l'administration municipale d'éclairer à fond ces faits. »

(12) Ibid. 
est à notre connaissance que d'autres communes du district ont une opération inverse, tel par exemple du quart de la fortune réelle » (13).

Aux prises avec les dysfonctionnements du système des pouvoirs locaux, submergée par une quantité chaque décade plus impressionnante de plaintes pour injustice fiscale, l'administration départementale faillit à sa mission technicienne, d'autant plus qu'elle ne peut compter sur l'établissement d'un véritable partenariat avec le pouvoir central parisien.

Les relations pouvoir départemental / pouvoir parisien ont un caractère unilatéral. Le pouvoir central décide, l'administration départementale exécute. «Il faut asseoir et percevoir rapidement. Voilà votre tâche » écrit le ministre des Finances à l'administration de l'Aisne (14). En retour, le pouvoir central parisien est fréquemment informé de l'évolution des travaux de répartition : les comptes rendus du commissaire au ministre des Finances sont envoyés une à deux fois par semaine. Lorsqu'un problème technique, voire juridique, surgit à l'occasion des opérations de l'emprunt, l'administration départementale s'adresse, elle aussi, aux ministres. Parfois s'instaure une tentative d'échange de vue entre les deux pouvoirs : le commissaire départemental plaidant à trois reprises pour un emprunt véritablement progressif afin de résoudre les injustices et de hâter son recouvrement (15). Cependant, si les pouvoirs départementaux sont unanimement soucieux de maintenir un lien informatif et consultatif auprès du pouvoir central, la réciproque n'est guère vraie. Rares sont les réponses du ministre des Finances aux demandes de renseignements ou aux appels à l'arbitrage en provenance du département. Qu'on en juge : lorsque à maintes reprises, les pouvoirs départementaux se font les porte-parole de la demande d'exemption des cantons dévastés par l'invasion autrichienne d'août-septembre 1793, ils ne reçoivent aucune réponse des ministres (16). Quant aux projets de réforme fiscale présentés par le commissaire Dormay pour limiter les inégalités, ils ont connu l'audience et le succès des papiers voués aux fonds de tiroir d'un bureau ministériel.

À la faveur des difficultés de répartition de l'emprunt et du recouvrement de ses premières classes, le lien politique entre pouvoir central / pouvoir départemental et pouvoirs locaux s'est distendu. Toutefois deux grandes décisions du pouvoir parisien posent les bases d'une redéfinition des rapports politiques au sein du système des pouvoirs nationaux, pour accroître l'efficacité de la levée d'un emprunt forcé qui est déjà un échec.

(13) Ibid., L 892, 27 pluviôse an IV, « Pétition au ministre des Finances de quarante et un habitants de Vervins en date du 2 pluviôse an IV. »

(14) Ibid., L 832. Lettre du ministère des Finances aux administrateurs du département le 24 nivôse an IV (14.01.96).

(15) Ibid., L 439. Adresses du commissaire départemental au ministre des Finances datées du 5 pluviôse, 26 nivôse an, 11 pluviôse an IV.

(16) Ibid., 11 ventôse IV (01.03.96). 
Pour éviter un fiasco, les conseils décident de relancer la levée de l'emprunt selon de nouvelles modalités beaucoup plus souples. La nouvelle loi du 26 pluviôse an IV (15 février 1796) met fin à l'égalité numérique des citoyens à l'intérieur de chaque classe et de nouveaux délais de recouvrement sont accordés (le terme de l'emprunt est fixé au 15 germinal an IV, soit le 4 avril 1796) (17). Certes, cette nouvelle loi répond à la recherche d'une plus grande justice fiscale sans se départir d'une exigence d'efficacité accrue dans la conduite des opérations de recouvrement. Mais, en contrepartie, elle alourdit considérablement le travail imputable aux pouvoirs locaux. Les rôles, si difficilement établis, sont désormais à refaire.

Un autre événement se produit le 28 pluviôse (17 février) en relation avec la réorganisation de la levée de l'emprunt forcé. L'administration départementale est destituée à l'initiative du Directoire sous le chef d'accusation que «... Les administrateurs ont profité de la loy sur l'emprunt forcé pour taxer arbitrairement les citoyens dans la répartition qu'ils ont faite, favoriser les riches et taxer outre mesure les propriétaires médiocres ; qu'ils ont d'ailleurs mis dans la confection des rôles une telle inertie, que le $16 \mathrm{du}$ présent mois, ils n'avaient encore envoyé que ceux des $16^{\mathrm{e}}, 15^{\mathrm{e}}, 14^{\mathrm{e}}$ classes... »(18). Épargner les plus riches citoyens du département en les déplaçant dans une classe inférieure à celle correspondant à leur fortune au détriment de citoyens plus modestes, dont la fortune a été surestimée de manière à ce qu'ils figurent dans des classes élevées de l'emprunt, n'est en rien une pratique abusive exceptionnelle. Le mode de répartition en classe, visant uniformément le quart des contribuables dans chaque canton permettait de telles dérives iniques tant à l'échelle des citoyens qu'à celle des cantons. Robert Schnerb avait déjà constaté dans le Puy-de-Dôme, lors des opérations de ce même emprunt forcé de l'an IV, que les cantons de la Montagne, plus pauvres, étaient beaucoup plus imposés proportionnellement que ceux de la Limagne pourtant plus riches (19).

Mais comment le pouvoir central parisien fut-il averti de telles irrégularités commises par l'administration départementale de l'Aisne ? En premier lieu, les rapports du commissaire départemental, dont les motifs d'affrontement avec l'administration départementale se sont multipliés à l'occasion d'affaires plus politiques, ont alerté les ministres, tout autant d'ailleurs, que les maladresses de l'administration départementale. À de multiples reprises, celle-ci avait fait part au ministre des Finances du manque de «grosses fortunes » dans le département pour remplir les cinq classes les plus imposées selon les normes légales. Plus que circonspect, supposant un mauvais

(17) Ibid., L 375,29 ventôse an IV (19.03.96).

(18) Ibid., L 301, le 21 ventôse an IV.

(19) Robert SCHNERB, Les contributions directes à l'époque de la Révolution dans le département du Puy-de-Dôme, Paris, Félix Alcan, 1934, pp. 344-347. 
gré fiscal et l'alliance maintes fois réaffirmée des notables et d'une administration qui ne pouvait en être que leur émanation par le jeu du scrutin censitaire, le ministre Faipoult lui répondit dans un post-scriptum cinglant : «Vous remarquerez que dans votre département les fermiers enrichis par beaucoup de circonstances peuvent entrer dans vos $15^{\mathrm{e}}$ et $14^{\mathrm{e}}$ classes » (20). Les notables qui composaient cette administration étaient trop tentés d'épargner leurs électeurs et homologues. Enfin, les malversations fiscales remontent jusqu'aux yeux et aux oreilles du ministre, pas seulement par l'intermédiaire du commissaire, mais aussi par celui des citoyens. Il n'est pas rare que ces derniers, déboutés de leur plainte contre la répartition de l'emprunt par l'administration départementale, en référent directement au ministre, "court-circuitant" en quelque sorte le système informatif institutionnel. Par exemple, l'adresse des quarante et un citoyens vervinois a été directement envoyée au ministre, qui l'a refusée au motif que les adresses en nom collectif étaient prohibées. Or cette adresse désignait ouvertement l'administration départementale comme l'une des responsables des injustices fiscales (21).

Cette réorganisation des pouvoirs locaux aboutit-elle à une plus grande efficacité fiscale dans le département ? À première vue, les nouvelles opérations de répartition de l'emprunt gagnent en rapidité puisque le 21 prairial an IV (9 juin 1796), soit quatre mois après la loi de réorganisation de la levée, les nouveaux rôles fiscaux sont totalement achevés (22). Le département figure dans la moyenne nationale puisque, selon les observations de Georges Lefebvre, la plupart des rôles en France n'ont été achevés qu'au mois de juillet (23). Le 12 messidor an IV (30 juin 1796), la moitié du montant total de l'emprunt a été recouvrée (24). Mais ce regain d'efficacité ne doit pas masquer la permanence des solidarités locales face à l'État fiscal. Effectivement, la nouvelle administration départementale profite de la loi fiscale du 26 pluviôse pour diminuer de moitié le montant de l'emprunt forcé qui lui était initialement imputé (25). Rien d'original ni de subversif dans cette opération, qui semble assez répandue puisque dans le Puy-de-

(20) ADA, L 892. Lettre du ministre des Finances aux administrateurs du département, le 19 nivôse an IV.

(21) Ibid, 27 pluviôse. Pétition de 41 citoyens de Vervins, en date du 2 pluviôse «... Que par l'opération générale du département qui a fait sauter les citoyens de la $3^{\mathrm{e}}$ classe à la $7^{\mathrm{e}}$ et ceux de la $7^{\mathrm{e}}$ à la $15^{\mathrm{e}}$, des fortunes de 10000 livres payeraient comme 30000 1, celles de 500001 comme celles de 200000 et $3000001 \ldots \gg$.

(22) Ibid., L 440, 21 prairial an IV. Le commissaire départemental au ministre des Finances:

(23) Georges LEFEBVRE, La France sous le Directoire. 1795-1799, Paris, Messidor, coll « Terrains », réed 1984, p. 124.

(24) ADA, L 892,12 messidor an IV. Sur un montant de $6105510 \mathrm{~F}$ attendus au titre de l'emprunt forcé, $3268051 \mathrm{~F}$ ont été perçus.

(25) Ibid., L440,21 prairial an IV. 
Dôme l'administration départementale, à la faveur de la même loi, était parvenue à réduire le sien des deux tiers (26).

Cependant cette résistance fiscale provoque une nouvelle disjonction entre les deux pouvoirs départementaux. Le commissaire Dormay avertit le ministre des Finances des agissements frauduleux de la nouvelle administration départementale. Non seulement la nouvelle répartition effectuée par les administrateurs départementaux comporte davantage de citoyens taxés pour un montant moindre, mais en plus, elle repose, une fois encore, sur les citoyens les plus pauvres au profit des plus fortunés (27). Les nouveaux administrateurs, comme leurs prédécesseurs sont accusés d'iniquité et d'arbitraire. Le conflit entre les deux têtes du pouvoir départemental éclate de nouveau et pose le pouvoir central parisien en arbitre. Le ministre des Finances, dans une missive du 7 thermidor an IV (25 juillet 1796) désavoue le travail de l'administration départementale (28). Le 24 thermidor (11 août), une réponse unanime des administrateurs justifie leurs actions et porte sur un terrain strictement politique le conflit qui les oppose au commissaire (29). La dialectique partisans/opposants au régime est de nouveau utilisée pour éliminer l'adversaire : le commissaire Dormay est alors dépeint comme un intriguant qui «serre son parti»; parti tôt amalgamé aux «anarchistes» par des administrateurs «amis du gouvernement » (30). Le conflit trouvera son épilogue après le coup d'État du 18 Fructidor an $\mathrm{V}$ avec la destitution de l'administration départementale (31).

Confrontée à la gestion de la crise politique du canton de Bohain, l'administration départementale choisit-elle un règlement local ou s'en réfèret-elle au pouvoir central ? Dans ce dernier cas, la circulation de l'information au sein du système des pouvoirs devient un enjeu politique majeur.

Les mois de vendémiaire et brumaire an IV sont un peu dans le canton de Bohain, ce que le mois de thermidor an II fut à la nation : une revanche, au caractère parfois réactionnaire. Dans ce canton du Vermandois d'environ 13181 habitants (32), étroitement intégré dans l'aire proto-industrielle du

(26) Robert SCHNERB, op. cit., pp. 346-347.

(27) ADA, L 440, 21 prairial an IV, le commissaire départemental au ministre des Finances : «Les nouveaux rôles de l'emprunt forcé sont enfin achevés, ce que je vous avois précédemment annoncé s'est réalisé, leur produit monte à 6105510 francs au lieu de 12109900 produit des anciens; le nombre des contribuables monte à 26595 dans les nouveaux rôles au lieu de 22663 qui se trouvaient imposés dans les anciens [...] Cet emprunt pèse plus particulièrement sur les malheureux qui ne pourront l'acquitter... ».

(28) Ibid, L 892, 7 thermidor an IV, lettre du ministre des Finances à l'administration départementale de l'Aisne.

(29) Ibid, 24 thermidor an IV.

(30) Ibid

(31) AN, $\mathrm{F}^{1 \mathrm{~b}}$ II Aisne 2, 4 vendémiaire an VI.

(32) Selon le recencement de l'an V. Chiffre repris dans tous les rapports officiels. 
complexe textile saint-quentinois, la première manifestation «thermidorienne » se déroule lors de l'installation, en brumaire an IV, de la nouvelle municipalité irrégulièrement élue. Les anciens administrateurs républicains sont insultés des noms et quolibets de «Robespierre, terroristes et buveurs de sang », puis frappés lors de l'assemblée électorale. L'ancien maire de la commune, en fonction avant l'an II - Vincent Leroy - est invité par ses anciens administrés à rentrer d'émigration et à reprendre ses fonctions municipales (33). L'opération s'effectue au détriment des perdants des élections irrégulières : les républicains ou «patriotes de $89 »$ comme ils s'intitulent. Regroupés derrière Louis Delassus, maire de l'an II à brumaire an IV, ils s'organisent et font arrêter par la gendarmerie de Saint-Quentin, Vincent Leroy, à son retour à Bohain. Cette arrestation sert de détonateur à l'explosion des tensions politiques internes au canton. Les actes contre-révolutionnaires, de l'arbre de la liberté abattu (34) au rappel des émigrés en passant par la réinstallation festive d'un prêtre réfractaire dans son presbytère jadis transformé en école (35), se multiplient. Cette violence culmine en nivôse an IV avec l'agression physique par un partisan de Vincent Leroy, de Louis Delassus l'ex-maire républicain nommé depuis, en frimaire an IV, commissaire cantonal (36).

Dès lors, l'enjeu local pour le pouvoir départemental est le rétablissement de l'ordre. Cela passe en priorité par le contrôle politique du canton. Celui-ci se fera-t-il avec ou sans le concours de l'État central?

L'État, par l'intermédiaire des pouvoirs départementaux, bénéficie de deux opportunités pour intervenir dans les affaires plutôt troublées de ce canton. La première est le procès pour émigration de Vincent Leroy, ancien maire, émigré lors de l'invasion autrichienne d'août-septembre 1793, de retour à Bohain en brumaire an IV après que la nouvelle municipalité de canton l'ait rappelé pour se mettre à sa tête. Le verdict de ce procès, s'il aboutit à la proscription de l'inculpé, peut décapiter le parti contre-révolutionnaire à Bohain. La seconde opportunité d'intervention est offerte par «l'attentat» (comme les républicains le désignent) perpétré sur le commissaire et ancien maire républicain Delassus par un partisan de Vincent Leroy. Elle peut permettre la destitution de la nouvelle municipalité peu acquise au régime.

Vincent Leroy, arrêté à son retour d'émigration, plaide sa radiation de la liste des émigrés, arguant du bénéfice d'une exemption de la loi du $4^{\mathrm{e}}$ jour complémentaire an III. Cette exemption stipule que certains émigrés

(33) AN, $\mathrm{F}^{\mathrm{lb}}$ II Aisne 9, 19 brumaire an IV. Adresse de la municipalité sortante aux Cinq-Cents.

(34) ADA, L 439, 11 nivôse an IV.

(35) AN, $F^{1 b}$ II Aisne 9, 19 brumaire an IV.

(36) Ibid., 28 pluviôse an IV. 
peuvent être rayés des listes de proscription s'ils justifient d'un état social de « cultivateurs et ouvriers ». Pour en obtenir le bénéfice, huit citoyens doivent témoigner en sa faveur en présentant une déposition écrite précisant la qualité sociale de l'inculpé et certifiée conforme par la municipalité et son commissaire. Une bonne partie des Bohainois s'efforce de réunir, ou plutôt de fabriquer ces pièces justificatrices. Le commissaire républicain Delassus s'y oppose puisque Vincent Leroy ne peut répondre de la qualité de «cultivateur », mais de celle de «bourgeois et marchand de bois». Il revient à l'administration départementale de se prononcer sur cette affaire. Dans un premier temps, pendant presque deux mois, du 25 frimaire an IV (16 décembre 1795) au 21 pluviôse an IV (10 février 1796), elle tergiverse, esquivant la prise de décision, tantôt par l'absence de l'un de ses membres (37), tantôt en refusant comme entachées de falsifications les pièces présentées pour la défense de Leroy (38). Puis, dans un second temps, le 21 pluviôse, à une majorité de trois voix contre deux, les cinq administrateurs départementaux reconnaissent la qualité de cultivateur à Vincent Leroy et par conséquent le rayent de la liste des émigrés en dépit des soupçons de faux qui pèsent sur les justificatifs fournis à l'appui (39). Le commissaire central, avec le soutien des deux administrateurs qui s'étaient opposés à cette décision, refuse un tel jugement et en intercède auprès des ministres de l'Intérieur et de la Justice. Il est vrai que le commissaire informait constamment les ministres des moindres évolutions de l'affaire Vincent Leroy. Ces affrontements intra-pouvoirs départementaux profitent à l'État central parisien. C'est avec l'aval des ministres que le commissaire départemental peut prendre une série de mesures contraires aux décisions de l'administration départementale, à commencer par le maintien en détention de Vincent Leroy (40). Quant aux maire et adjoints de Bohain, ils sont arrêtés pour crime de faux : sur les rôles qu'ils avaient présentés à l'administration départementale, ils avaient biffé la qualité de «bourgeois » accolée au nom de Vincent Leroy pour lui apposer celle de «cultivateur» (41). Le 21 thermidor (8 août 1796), un arrêté du Directoire exécutif les suspend de leurs fonctions (42).

Preuve de l'efficacité du soutien de l'État à son commissaire départemental en conflit avec les pouvoirs élus, le 28 pluviôse an IV (17 février 1796), quinze jours après qu'elle ait rendu son verdict sur l'affaire Leroy, l'administration départementale est destituée. L'acte de destitution dressé

(37) ADA, L 439, 21 pluviôse an IV.

(38) Ibid., 17 pluviôse an IV.

(39) Ibid., 21 pluviôse an IV.

(40) Ibid.

(41) Ibid., 7 germinal an IV.

(42) AN, F ${ }^{1 b}$ II Aisne 9, 14 nivôse an V. 
par le Directoire invoque comme motifs de sa décision, la protection accordée par l'administration aux émigrés rentrés, son manque de soutien aux commissaires du pouvoir exécutif et sa répartition inique de l'emprunt forcé dans le but de « favoriser les riches » (43).

Le procès de Vincent Leroy favorisa l'affrontement du personnel politique local puis l'implosion des pouvoirs départementaux. Sur l'enjeu de la reconnaissance du statut d'émigré à Leroy, se sont déployées, d'une part, une division désormais classique entre le commissaire départemental Dormay et l'administration départementale, d'autre part, une autre, plus originale, entre les membres de cette même administration. Cet éclatement du pouvoir départemental profita une fois de plus à l'État central, désormais en situation d'arbitrage et de recours pour des commissaires départemental et cantonal en proie aux oppositions des pouvoirs élus.

La seconde opportunité offerte à l'État central et à son appareil administratif pour intervenir dans les affaires du canton de Bohain, confirme le précédent constat d'un effacement de «l'esprit local». Une première fois déboutés par l'administration départementale pour présentation de documents non visés par le commissaire cantonal et par conséquent illégaux, les huit témoins bohainois qui cherchent à justifier du prétendu état de cultivateur de Vincent Leroy, se trouvent dans une impasse. Comment faire approuver des faux documents par le commissaire cantonal, et républicain convaincu, Louis Delassus, le pire ennemi de leur protecteur? C'est alors que le 30 nivôse (20 janvier 1796), devant son refus réitéré d'apposer sa signature sur de faux certificats en faveur de Vincent Leroy, le commissaire Delassus est frappé par un dénommé Thomas Leroux (44).

À propos de cette nouvelle affaire, le commissaire départemental entend privilégier l'intégration du local au national plutôt que la recherche d'un accommodement local. Autrement dit, l'axe Paris / commissariat départemental est exclusivement favorisé aux dépens de celui qui relie le commissariat départemental au commissariat cantonal. Cette stratégie permet de comprendre pourquoi dans le conflit bohainois, l'imposition stricte de la loi a toujours été privilégiée sur les tentatives de règlements locaux pourtant défendus par la totalité des Bohainois, au-delà de leurs clivages politiques.

Par exemple, après l'agression du commissaire, une entreprise de conciliation entre agresseur et agressé est conduite par la municipalité de Bohain sous les auspices du juge de paix (45). La victime accepte la médiation, pardonne et inflige une punition républicaine symbolique à son agresseur.

(43) ADA, L 439, 7 ventôse an IV.

(44) Ibid., 14 pluviôse an IV.

(45) AN, F ${ }^{1 \mathrm{~b}}$ II Aisne 9, Bohain, 4 pluviôse an IV. 
Le commissaire cantonal Louis Delassus informe alors son supérieur Joachim Dormay, commissaire départemental, de son choix d'un règlement local. Il le justifie dans le but de «ramener la paix dans le canton» et, en conséquence lui enjoint de ne pas intervenir, afin de ne pas raviver les tensions (46). Mais le commissaire départemental n'en a cure : il avertit le ministre de la Police générale et décide, de concert avec lui, de toute une série de mesures de représailles contre l'agresseur (47). Un détachement de gendarmerie est envoyé dans le canton (48). Quant à l'agresseur, il est arrêté, au mépris des gages que lui avait donnés le commissaire (49).

Dès lors, le canton entre dans un conflit ouvert avec l'État et ses représentants. Le commissaire Delassus, désormais considéré comme traitre à sa parole, concentre toutes les haines. En la personne du commissaire, c'est tout l'État directorial qui est discrédité. Sa personne, interface incontournable pour la définition et la modulation des liens politiques et sociaux entre les citoyens du canton de Bohain et l'État, devient l'incarnation de la perte de légitimité d'un régime qui n'hésite pas à bafouer sa Constitution pour se maintenir. En privilégiant la loi et les liens qui l'unissent au pouvoir central parisien au détriment de ses relations interpersonnelles avec la société locale, le commissaire départemental a plus que jamais affaibli, pour ne pas dire compromis, l'intégration politique du canton de Bohain à l'État directorial. Un désordre endémique perdurera dans ce canton jusqu'au Consulat.

Avant fructidor an $\mathrm{V}$, le champ politique du département est parcellisé. Il n'est qu'un espace d'affrontements entre pouvoirs locaux rivaux où chaque mission à accomplir devient un enjeu de pouvoir. Ce contexte de rivalités internes présentes à tous les niveaux des pouvoirs locaux favorise la prééminence des liens verticaux de domination entre les ministres et les commissaires aux dépens des liens horizontaux de solidarité entre pouvoirs locaux. Mais, une fois encore, cette suprématie des liens d'ordre verticaux émanant des personnels de l'État central rencontre ses limites. Elle ne fonctionne plus lorsqu'elle est confrontée au dilemme : auto-gouvernement ou respect intégral local de la règle élaborée par le centre parisien ? C'est, en somme, tout l'esprit local qui s'efface au profit de la présence de l'État lorsque la détention et la délégation des pouvoirs locaux sont variées, contradictoires, voire antagonistes. À champ politique local fragmenté, État fort. Mais, la volonté d'uniformisation des personnels politiques locaux consécutive au coup d'État du 18 Fructidor an V bouleverse-t-elle profondément ces paramètres?

(46) Ibid., procès-verbal du commissaire de Bohain, 4 pluviôse an IV.

(47) Ibid., 28 pluviôse an IV.

(48) Ibid.

(49) Ibid. 


\section{Circulation de l'information et prise de décision politique sous le Second et le Troisième Directoire. L'exemple de l'an VII.}

Il est quelque peu arbitraire de séparer un Premier d'un Second Directoire en fonction de l'événement constitué par le coup d'État du 18 Fructidor an V (4 septembre 1797), surtout en ce qui concerne l'histoire des rapports entre pouvoirs locaux et État central puisqu'en amont comme en aval de cette date bien des continuités se sont déployées. Cependant, dans maints départements, à commencer par celui de l'Aisne, le coup d'État du 18 Fructidor s'est traduit par une homogénéisation du personnel politique local. À la parcellisation du champ politique de l'an IV et de l'an V succède donc, dès l'an VI, une plus forte unité. En suscitant une vague d'épuration du personnel local, le coup d'État du 18 Fructidor an V a permis que toutes les fonctions locales d'importance soient fermement reprises en main par des «républicains directoriaux» (50). Si la coopération entre personnel nommé et personnel élu est désormais facilitée, cela signifie-t-il pour autant une recomposition du rapport local / national ? En uniformisant le personnel politique local pour le réduire à ses seuls partisans, le pouvoir central ne s'est-il pas départi d'un pouvoir de contrôle de la vie politique locale? Une telle hypothèse mérite d'être posée pour l'an VII, si l'on se souvient que les affrontements politiques locaux de l'an IV avaient eu pour conséquence d'accentuer le contrôle politique et social de l'État sur la société locale.

La levée d'un nouvel emprunt forcé en l'an VII constitue une situation privilégiée pour mesurer la capacité des pouvoirs locaux à exécuter les directives du pouvoir central et l'évolution de leur attitude face au devoir fiscal, seulement trois ans après un précédent exercice difficile.

D'emblée, il apparaît que la levée de l'emprunt de l'an VII fut beaucoup moins difficile et accaparante pour l'administration départementale de l'Aisne que celui de l'an IV. Cette situation résulte essentiellement de la nouvelle politique fiscale du ministre Ramel. D'une part, les exigences de l'État paraissent plus réalistes qu'en l'an IV : de l'emprunt forcé voté le 19 thermidor an VII (6 août 1799), le Directoire attend 100 millions et non plus 600 millions comme en l'an IV Mais il est vrai que ce nouvel emprunt forcé a pour priorité de financer l'effort de guerre, à la différence de son prédécesseur de l'an IV qui, lui, devait épurer le papier-monnaie en circulation. D'autre part, la lente émergence d'un État fiscal se traduit par la mise en place d'une administra-

(50) Ibid., «Dossier an VI », 4 vendémiaire an VI : propositions de personnel par le commissaire départemental après les destitutions de l'administration centrale de l'Aisne et des municipalités de Laon et Saint-Quentin. 
tion fiscale plus efficace. Les administrations départementales sont soulagées d'une grande partie des travaux de répartition et de recouvrement par la formation de deux jurys. L'un, dit de taxation, composé des administrateurs et de citoyens désignés, est chargé de la répartition de l'emprunt; l'autre, dit de révision, où ne siégent que des contribuables, doit examiner les réclamations. Enfin, le principe de progressivité, auquel s'étaient refusés en l'an IV la plupart des députés, est désormais institué.

Cependant la levée de l'emprunt forcé dans le département de l'Aisne, quoiqu'organisée dans des conditions beaucoup plus favorables, fut tout aussi lente que lors de l'exercice de l'an IV. À maintes reprises, le ministre des Finances se plaint du retard de l'administration départementale dans l'exécution des opérations fiscales qui lui incombent (51). Ainsi, le 26 vendémiaire an VIII (18 octobre 1799), soit trois mois après le vote de l'emprunt, un cinquième rapport de Robert Lindet, ministre des Finances du Directoire, rappelle que l'Aisne figure toujours parmi les trente-six départements qui ne disposent pas encore de leurs tableaux de taxation (52). Avec trois jours de retard, le 29 vendémiaire an VIII (21 octobre 1799), le jury de taxation du département remet enfin ses travaux : ils font état de 1303 prêteurs pour un montant estimé à 1255509 francs (53). Alors comment expliquer cette lenteur?

Comme en l'an IV, la résistance fiscale doit être invoquée. Il semblerait qu'avant de présenter ses résultats au pouvoir central, le jury de taxation cherche à gagner du temps afin de ménager les intérêts locaux. Ce mauvais gré est relayé par l'administration départementale. Le 29 vendémiaire an VIII, elle informe le ministre des Finances que les résultats définitifs de la taxation - les précédents étant sujets à une révision du jury - ne lui parviendraient que le 11 brumaire (2 novembre 1799), au plus tard (54). Or, il n'en eut connaissance que le 24 brumaire (15 novembre) (55). Entre ces deux dates, jamais l'administration départementale n'a fait pression sur le jury, en dépit des remontrances réitérées du pouvoir parisien (56). Mais,

(51) ADA, L 894, dossier «pièces diverses». «Le jury de taxation qui devait suivant la loi du 19 thermidor finir son travail en 10 jours, et en un mois au plus, suivant la loi du 6 fructidor n'a pas achevé ses opérations dont vous m'avez même laissé entièrement ignorer les progrès depuis le 27 fructidor dernier » écrit le ministre des Finances, le 17 vendémiaire an VIII, à l'administration départementale.

(52) Corps législatif, Procès-verbaux du conseil des Anciens. Vendémiaire an VIII, Paris, Imprimerie nationale, non daté.

(53) ADA, L 894, 29 vendémiaire an VIII.

(54) Ibid.

(55) Ibid., 24 brumaire an VIII.

(56) Ibid., 17 brumaire an VIII. Le message lapidaire de Robert Lindet aux administrateurs départementaux en date du 7 brumaire est représentatif de la résistance fiscale des pouvoirs locaux : «J'attends de jours en jours les copies des taxes à l'emprunt forcé de 100 millions que je vous ai demandées par ma lettre du 25 thermidor dernier. Je vous ai plusieurs fois exprimé mon impatience pour la réception des copies dont il s'agit. Aujourd'hui, 7 brumaire, elles ne me sont pas encore parvenues. Rien ne peut plus justifier un tel retard... »; à titre de comparaison, les travaux du jury sont achevés dans le Puy-de-Dôme le 23 vendémiaire an VIII (dans Robert SCHNERB, op. cil., pp. 542). 
entre ces deux dates, s'est accompli le coup d'Etat de Bonaparte et Sieyès. Les pouvoirs locaux axonais ont-ils cherché à profiter de ce coup de grâce porté au Directoire pour abandonner la charge fiscale qui pesait sur les plus fortunés d'entre eux? Dans ce cas, pourquoi les autorités départementales ont-elles finalement rendu publics les résultats de la répartition le 24 brumaire an VIII ? Rien n'interdit de penser cet acte comme un premier signe tangible de ralliement des notables au nouveau pouvoir. Effectivement, ce ne fut qu'après quelques jours de tergiversations, voire de sourde résistance, que les autorités départementales proclamèrent officiellement le 27 brumaire (18 novembre) leur adhésion officielle au nouvel ordre politique issu du 18 Brumaire an VIII (57).

À la différence de l'exercice de l'emprunt forcé de l'an IV, celui de l'an VII n'a provoqué aucun dysfonctionnement dans le système des pouvoirs locaux. Au contraire, on observe même une certaine tendance à l'autonomisation du local par rapport au national. Les rapports de l'administration départementale et de son commissaire envoyés aux ministres sont aussi rares que les tensions entre toutes les composantes des pouvoirs locaux. Un large front du refus local associant la plupart des pouvoirs locaux, pourtant composés de parfaits « républicains directoriaux », s'est constitué contre le prélèvement fiscal initié par l'État central. Ce dernier n'a cessé de réclamer à l'administration départementale, de thermidor à vendémiaire an VII, des informations sur l'avancement des travaux de l'emprunt; rien ne lui a été retourné.

À la différence de ce qui se passait en l'an IV, cette perte de contrôle du local par l'État central se traduit par une plus grande difficulté à faire rentrer l'emprunt forcé. En l'an VII, la perspective d'un «État fiscal », pourtant en cours d'élaboration, s'est éloignée des citoyens axonais, par l'interposition du rôle des pouvoirs locaux solidaires (58). L'interface du pouvoir départemental, plus uni qu'auparavant, assume la quasi-totalité de ce contrôle politique et social local et se pose, non plus en une simple courroie exécutive des volontés de l'État central, mais plutôt en un indispensable partenaire. La décision centrale est désormais discutée localement pour être aménagée et redéfinie. Pourtant, à tous les échelons des pouvoirs locaux, le personnel politique fut, à une très écrasante majorité, désigné par le régime. Plus qu'en l'an IV, des pratiques politiques notabilitaires face à l'État central s'instaurent en l'an VII.

(57) Cf. Laurent BRASSART, « Réception et acceptation du coup d'État du 18 brumaire an VIII », dans Jean-Pierre JESSENNE et alii (éd.), Du Directoire au Consulat, t. 3, Brumaire dans l'histoire du lien politique et de l'État-Nation, Lille, 2001, pp. 173-194.

(58) Sur la problématique de la construction de l'État fiscal, cf. Claudine WOLIKOW, «Fiscalité et citoyenneté sous le Directoire ou la déroute de l'État sans fisc », dans Hervé LEUWERS et alii (éd.), op. cit., pp. 217-237. 
En l'an IV, l'État central, en s'appuyant sur le corps des commissaires, possédait des instruments de contrôle du canton de Bohain, notamment parce qu'il disposait d'une information précise et fréquente. En l'an VII, les pouvoirs locaux sont-ils toujours en mesure de faire circuler l'information jusqu'au pouvoir central?

Les tensions politiques croissantes depuis l'an III font progressivement basculer le canton de Bohain dans l'anti-Révolution en l'an VII. On a vu précédemment que le refus par le commissaire départemental de tout règlement local des problèmes inhérents au canton en l'an IV n'avait fait que précipiter une telle évolution. Depuis, tous les caractères de l'antiRévolution y ont libre cours : refus de l'impôt, inapplication du calendrier révolutionnaire et des fêtes républicaines, insoumission à la conscription, pratique ostentatoire du culte catholique (59). Cette situation est aggravée par le dysfonctionnement des pouvoirs cantonaux. Bien que renouvelée, la municipalité cantonale reste très hostile aux républicains directoriaux regroupés derrière l'inamovible commissaire Louis Delassus. En l'an VII, l'enjeu politique polarisateur des passions est la réintroduction de la présence de l'État au sein du local, à moins que ce ne soit la nécessaire réintégration d'un canton devenu quasi autonome au sein de l'État-Nation. Pareil processus passe au préalable par l'exécution des lois dans le canton.

Croyant bénéficier, comme en l'an IV, d'un appui sûr auprès de son supérieur hiérarchique le commissaire départemental, le commissaire du canton de Bohain, Louis Delassus lui adresse des rapports alarmistes afin de le presser à agir contre la municipalité de Bohain. Mais, depuis l'an IV, bien des paramètres politiques ont changé ; Joachim Dormay commissaire départemental en l'an IV a depuis été élu en germinal an VI à l'assemblée des Cinq-Cents; lui ont succédé au commissariat départemental, d'abord Quentin Duplaquet, avant qu'il ne rejoigne à son tour en germinal an VII le conseil des Cinq-Cents, puis, Jean-Baptiste Martin, un ami proche du député Jean Debry (60). De plus, si en l'an IV, le commissaire Dormay était confronté à un conflit permanent avec l'administration départementale élue, tel n'est plus le cas en l'an VII ; désormais entre l'administration et son commissaire, il y a plus qu'une communauté de vue et d'intérêt : tous sont de bons « républicains directoriaux ».

C'est pourquoi, de brumaire à messidor an VII, les pouvoirs départementaux choisissent la non-intervention dans les affaires intérieures du canton de Bohain. Non seulement, ils ne répondent pas aux requêtes du commissaire cantonal, mais en plus ils n'hésitent pas à le désavouer dans son

(59) Cf. Laurent BRASSART, DU Directoire au Consulat, le département de l'Aisne. Crise et transition politique du 18 fructidor an V au 26 messidor an IX, Mémoire de DEA d'histoire, Université de Rouen, mai 1999, pp. 133-143.

(60) AN, $\mathrm{F}^{\mathrm{lb}}$ I 103. Nomination de Quentin Duplaquet, le 28 germinal an VI. Nomination de Martin le 5 prairial an VII. 
conflit avec la municipalité (61). Évidemment, de frimaire à messidor an VII, les pouvoirs départementaux se gardent bien d'informer le pouvoir central parisien de la situation du canton de Bohain (62). Ces hommes, auxquels le Directoire a délégué tous ses pouvoirs, construisent les assises hégémoniques de leurs pouvoirs locaux. Il convient en quelque sorte de ne pas détruire le pacte tacite qu'ils ont passé avec le pouvoir central : assurer à la fois le calme politique à l'échelle du département et l'exécution efficace des directives de l'État en échange de la délégation des pouvoirs locaux. Bien évidemment, exposer la situation de Bohain au ministre reviendrait à remettre en cause un tel notables' agreement. Grand est donc l'isolement du commissaire de Bohain, aux prises avec un canton où aucune loi de la République ne semble s'appliquer.

En messidor an VII, l'administration départementale et son commissaire interviennent brutalement dans le conflit bohainois où ils font preuve désormais d'une intransigeance inaccoutumée à l'égard de la municipalité hostile au Directoire. Ainsi, le 6 thermidor an VII (24 juillet), elle suspend quatre agents et le secrétaire de la municipalité cantonale de Bohain (63). En réaction à l'inapplication des lois dans le canton par la municipalité, elle ordonne à la gendarmerie, le 22 fructidor (8 septembre 1799), de procéder à des visites domiciliaires contre les insoumis (64). Le 25 fructidor (11 septembre), elle invalide la réélection du secrétaire de la municipalité cantonale (65).

Un tel retournement, aussi brutal soit-il, ne peut se comprendre sans constater que le système informatif des pouvoirs locaux a été contourné par le commissaire cantonal de Bohain. Désavoué par son supérieur hiérarchique - le commissaire départemental - dans son combat contre la municipalité de Bohain le 18 germinal (7 avril 1799), le commissaire cantonal décide d'en référer directement aux ministres de l'Intérieur et de la Police générale. Bien lui en a pris puisque jamais les pouvoirs départementaux n'avaient averti les ministres de la situation antirévolutionnaire du canton de Bohain. Les rapports du commissaire ne dépassaient jamais le niveau du pouvoir départemental (66).

S'instaure la formation d'un axe informatif entre commissaire cantonal et pouvoir central parisien apte à contourner et influencer le niveau intermédiaire du pouvoir départemental. Aussi le 21 prairial ( 9 juin) et le 5 messidor (23 juin), le ministre de la Police intervient pour réclamer des

(61) ADA, L 461.12 germinal VII.

(62) Aucun rapport des pouvoirs départementaux aux ministres concernant le canton de Bohain n'a été retrouvé au cours de cette période tant dans les fonds des AN que dans ceux des ADA.

(63) ADA, L 315, 6 thermidor an VII.

(64) Ibid., L 314, 22 fructidor an VII.

(65) Ibid., L 315, 25 fructidor an VII.

(66) Constat établi après consultation des séries aux AN, F 1 BII Aisne II «Bohain », F lcIII Aisne 9, et aux ADA, L 448 et L 561, L 562, L 563. 
renseignements sur l'état du canton au commissaire départemental et l'invite en conséquence à « appeler son attention sur le canton» (67), « suite à une lettre publique d'un des fonctionnaires de votre arrondissement » (68).

Cette remodulation des liens institutionnels entre pouvoir central et pouvoirs locaux aboutit à briser la chape de plomb que les notabilités directoriales siégeant au pouvoir départemental avaient pris soin de poser sur le canton de Bohain. Le contrôle des pouvoirs départementaux sur la circulation de l'information des cantons vers le pouvoir parisien est pris en défaut. Le rétablissement de l'ordre dans le canton de Bohain commence et se réalise pleinement en vendémiaire an VIII (69).

En l'an VII, le personnel politique du département offre une forte homogénéité politique aux yeux du pouvoir parisien. À aucun moment, la stabilité politique de l'Aisne ne paraît compromise : depuis fructidor an V, les assemblées électorales n'envoient aux corps législatifs que de bons républicains directoriaux (70). L'an VII n'a pas failli à la règle. Le Directoire, assuré de l'issue favorable du scrutin, n'avait d'ailleurs pas accordé d'attention particulière à la préparation des élections dans le département (71). Tout ce personnel politique départemental relève des grands réseaux constitués depuis fructidor an $\mathrm{V}$ et centrés autour des députés les plus influents du département, à commencer par Jean Debry. Aucune fonction politique départementale d'importance en l'an VIL n'échappe à un de leur client. On observe donc une territorialisation accrue de la fonction de député. Tout semble fonctionner en l'an VII, comme si l'État central, pour prix de la stabilité politique du département, avait consenti une aliénation de son pouvoir de contrôle du territoire local à un personnel qui lui est acquis. Or, l'accomplissement des fonctions techniciennes de l'État, telles que les péripéties des travaux de l'emprunt de l'an VII l'ont montré, n'a pas été amélioré pour autant. Quant à la transmission d'une information remontant du local vers Paris, sa circulation n'a gagné ni en rapidité ni en fréquence. La rétention de l'information par les pouvoirs départementaux lors de la crise de l'an VII qui secoue le canton de Bohain confirme ce constat d'échec de la re-formulation des liens politiques personnels entre pouvoir central et pouvoir local par le Second Directoire. Au contraire, on observe une distanciation du local par rapport au national en l'an VII dans l'Aisne. L'apparent calme politique du département et sa fidélité au régime, réaffirmée lors des élections de

(67) ADA, L 619, 21 prairial an VII.

(68) Ibid., 5 messidor an VII.

(69) Ibid., L 448, 11 vendémiaire an VII.

(70) Cf. Éric MALFOY, Les élections législatives dans le département de l'Aisne sous le Directoire, Mémoire de maîtrise, Université de Valenciennes, 1998, 94 p.

(71) Bernard GAINOT, 1799, un nouveau Jacobinisme? La démocratie représentative, une alternative à brumaire, Paris, Éd. du C.T.H.S., 2001, pp. 31-43. 
l'an VII, cachent non seulement une pérennité de conflits locaux mais surtout une stratégie du repli des notabilités politiques sur le local.

Dans le département de l'Aisne, département à la culture politique modérée, situé à moins d'une journée de transport de Paris, il est possible, au terme d'une comparaison entre l'application locale des ordres parisiens et la circulation de l'information du local vers Paris au cours de l'an IV et de l'an VII, de constater une évolution paradoxale des rapports entre État central et pouvoirs locaux. Plus les divisions inter et intra-pouvoirs locaux redoublent, plus le contrôle du territoire local par l'État central se renforce. Moins les affrontements politiques déchirent les pouvoirs locaux, moins l'État central est en mesure de faire exécuter localement ses décisions. Le conflit interne aux pouvoirs locaux, résultant le plus souvent de l'hétérogénéité politique du personnel, est de nature à poser l'État central en allié pour l'un des partis contre l'autre. Généralement, dans ce cas de figure, se déploie un solide axe vertical, fait d'autorité hiérarchique, reliant la chaîne des commissaires locaux aux ministres. C'est cet axe qui est mis en œuvre en l'an IV pour que les travaux de l'emprunt forcé et les incidents survenus à Bohain trouvent une issue favorable aux intérêts de l'État. En revanche, en l'an VII, l'homogénéité politique du personnel axonais, tout acquis au régime, entrave paradoxalement le contrôle de l'État central sur le département. Toute une logique de liens horizontaux de coopération à chaque niveau des pouvoirs locaux, qu'ils soient élus ou nommés, s'impose au détriment de l'axe vertical d'autorité en fonctionnement en l'an IV Dès les lendemains du coup d'État du 18 Fructidor an $\mathrm{V}$, en concordance avec l'essai de stabilisation du régime directorial, s'érige dans l'Aisne, un «véritable pouvoir local » qui cherche à s'autonomiser de l'État central. La délégation de la totalité des plus importants pouvoirs locaux par le pouvoir central aux « républicains directoriaux » axonais, dans le but de contrôler davantage le territoire local, a produit des résultats strictement inverses à ceux attendus. Des réseaux locaux de solidarité, reliant les députés aux élus des administrations départementales et municipales, se sont intercalés entre les ministres parisiens et les citoyens axonais ; ils privilégient l'élaboration de règlements locaux plutôt que l'application aveugle des décisions du centre. La rétention de l'information concernant l'agitation politique du canton de Bohain et la résistance voilée à l'emprunt forcé en l'an VII par les pouvoirs départementaux en sont les manifestions patentes.

Laurent BRASSART

Université de Rouen

2, passage Philippe-Auguste 75011 Paris

$\underline{\text { laurentbrassart@voila.fr }}$ 